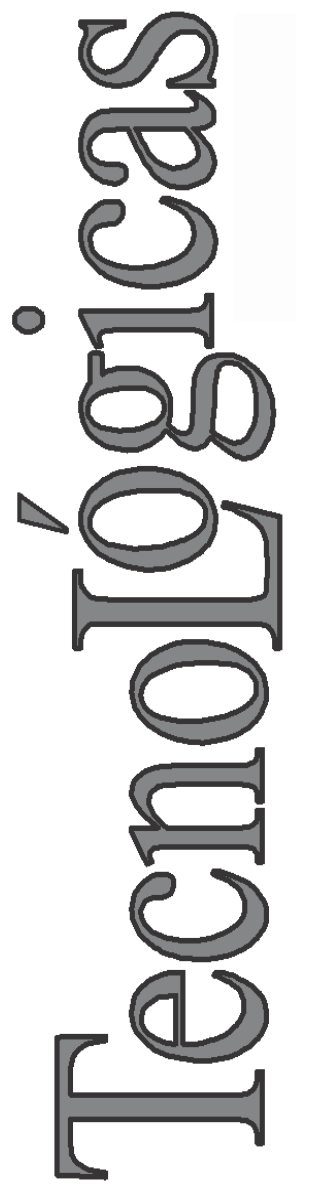

Artículo de Investigación/Research Article

Caracterización de Bordes y Contrastes en una Imagen Digital ante la Variación de Parámetros de los Filtros Pasa-Altos usados en la Estimación de Visibilidad Atmosférica

Characterization of the Edges and Contrasts in a digital image with the variation of the Parameters of the High-pass Filters used in the Estimation of Atmospheric Visibility

Martha C. Guzmán-Zapata ${ }^{1}$ Alejandro Restrepo-Martínez ${ }^{2}$

Recibido: 27 de mayo de 2013 Aceptado: 31 de julio de 2013

1 M.Sc. Automatización y Control Industrial, Facultad Ingenierías, Instituto Tecnológico Metropolitano, Medellín-Colombia marthaguzman5200@correo.itm.edu.co 2 Ph.D. Ingeniería - Sistemas, Facultad Ingenierías, Instituto Tecnológico Metropolitano, Medellín-Colombia alejandromartinez@itm.edu.co 


\title{
Guzmán \& Restrepo / Caracterización de Bordes y Contrastes en una Imagen Digital ante la Variación de Parámetros de los Filtros Pasa-Altos usados en la Estimación de Visibilidad Atmosférica
}

\section{Resumen}

En este documento se consideran los bordes y los contrastes obtenidos con los filtros pasa-altos que se utilizan en la estimación de la visibilidad atmosférica diurna desde imágenes digitales, cuyo comportamiento es caracterizado con la variación de los parámetros correspondientes a filtros pasa-altos tales como: el Ideal, Gaussiano, y Homomórfico-Gaussiano. Una imagen sintética que contiene regiones con distintos contrastes se utiliza para aplicar los diferentes filtros; luego, se define un índice para medir la calidad de los bordes obtenidos en las imágenes filtradas y se emplea para analizar los resultados. Los resultados muestran que tanto la selección del filtro como la selección de sus parámetros, afectan las características y la calidad de los bordes detectados en la imagen filtrada, también determinan la cantidad de ruido que el filtro agrega a la imagen (artefactos que no estaban presentes en la imagen original) y además establecen si se logra o no, la detección de los bordes; los resultados obtenidos también evidencian que el índice de calidad de los bordes alcanza valores máximos con ciertas combinaciones de los parámetros de los filtros. Esto significa que algunas combinaciones de parámetros disminuyen las situaciones que distorsionan los bordes y que distorsionan las medidas de visibilidad atmosférica basadas en la transformada de Fourier. Así que, estos parámetros, que proporcionan máximos de calidad para los bordes, se establecen como convenientes para ser utilizados en las medidas de visibilidad.

\section{Palabras clave}

Filtros pasa-altos, detección de bordes, visibilidad atmosférica, calidad de imagen, contrastes.

\begin{abstract}
This paper considers the edges and contrasts obtained with high-pass filters used in the estimation of daytime atmospheric visibility from digital images, and the behavior of these edges and contrasts is characterized by varying the parameters of high-pass filters such as the Ideal, Gaussian, and HomomorphicGaussian. A synthetic image of regions with different contrasts is used to apply different filters, then, we define an index to measure the quality of the edges obtained in the filtered image and it is used to analyze the results. The results show that both, the filter selection and the selection of its parameters: affects the characteristics and quality of the detected edges in the filtered image, also determine the amount of noise that the filter added to the image (artifacts that were not present in the original image), and also establish if achieved, or not, the edge detection. The results also show that the edge quality index reaches maximum values at certain combinations of the filters parameters, which means that some combinations of parameters reduce situations distorting the edges and distorting atmospheric visibility measures based on the Fourier transform. So these parameters which provide maximum quality edges are established as suitable for use in visibility measurement.
\end{abstract}

\section{Keywords} contrast.

High-pass filters, edge detection, atmospheric visibility, image quality, 


\section{INTRODUCCIÓN}

La medición de visibilidad atmosférica tiene aplicaciones en actividades tales como: la seguridad del tráfico aéreo y marítimo, la estimación indirecta de la contaminación del aire, y la estimación de la radiación solar (Xie et al., 2008). La visibilidad se mide de diversas formas: como rango visual (VR) equivalente a la mayor distancia a la cual un objeto negro puede ser reconocido cuando se observa contra el cielo, o como rango meteorológico (MR) definido como la distancia en la cual el objeto tiene un contraste de $2 \%$ respecto del fondo blanco (Caimi et al., 2004), también como rango óptico meteorológico (MOR) con un contraste de $5 \%$ (WMO-No 8, 2008), o se estima a través de índices de visibilidad correlacionados linealmente con las mediciones de VR, o MR, o MOR (Liaw et al., 2010).

Actualmente existe un interés por estimar cuantitativamente la visibilidad atmosférica diurna usando imágenes digitales; esta medición se realiza automáticamente reconociendo cambios bruscos en los valores de intensidad de gris (brillo) en una región de la imagen: por detección de bordes (Bäumer et al., 2008) y por medición de contrastes (Caimi et al., 2004). Entre las metodologías por detección de bordes están aquellas que extraen altas frecuencias espaciales con la aplicación de filtros pasa-altos sobre el espectro de Fourier de la imagen, para luego definir índices de visibilidad como el promedio de las intensidades de gris (o brillo) en la imagen filtrada que contiene los bordes (Luo et al., 2005; Sun et al., 2007); por lo que, la precisión de esta medida depende de la cantidad de bordes detectados y de sus características.

Según Sun et al. (2007) la precisión de esta medida depende del tipo de filtro, porque al utilizar un filtro pasa-altos del tipo ideal, en la imagen filtrada se observan artefactos adicionales los cuales no estaban presentes en la imagen original. Por lo que, el mismo grupo propone el uso de un filtro pasa-altos HomomórficoGaussiano asegurando que se mejora la precisión de la visibilidad al eliminar esos artefactos de la imagen filtrada. Y muestran que las correlaciones entre las estimaciones de visibilidad usando el filtro Homomórfico-Gaussiano, y las medidas de visibilidad tomadas por observadores expertos, dependen de uno de los parámetros 
Guzmán \& Restrepo / Caracterización de Bordes y Contrastes en una Imagen Digital ante la Variación de Parámetros de los Filtros Pasa-Altos usados en la Estimación de Visibilidad Atmosférica

de dicho filtro: el radio de corte $(D o)$ mostrando diferentes valores de correlación en cada caso; sin embargo, no estudian cómo se ven afectadas las estimaciones de la visibilidad con la variación de los otros parámetros del filtro: $Y H, Y L, c$.

Ya que en los trabajos previos no se ha establecido el valor de los parámetros de los filtros pasa-altos (Yang, 2005), en este trabajo se propone evaluar el efecto que tiene sobre los bordes de una imagen digital, y sobre los contrastes, la variación de los parámetros $(D o, Y H, Y L, c)$ correspondientes a los filtros pasa-altos del tipo Ideal, Gaussiano, y Homomórfico-Gaussiano, utilizados en las estimaciones de visibilidad atmosférica; buscando determinar los parámetros que en la imagen filtrada: logran la detección de los bordes y mejoran las características de los mismos. Los valores de los parámetros de los filtros, así determinados, permitirán en trabajos futuros estabilizar la precisión de la medida de la visibilidad, al mejorar las características de los bordes y al evitar, o disminuir, la adición de artefactos a la imagen filtrada.

\section{METOdOLOGía}

El borde se define como el cambio rápido (en pocos pixeles) de los valores de intensidad en una región de la imagen. Es posible medir características del borde si se analizan las intensidades de los pixeles sobre una trayectoria trazada perpendicularmente al borde (ver Fig. 1). La relación entre la intensidad versus la posición relativa del pixel en la trayectoria se denomina perfil de borde. En la Fig. 1, se muestra un perfil donde pueden medirse descriptores tales como: alto, ancho, pendiente y ruido; según Töpfer \& Linss, 2007, la composición de cualquier conjunto de medidas de calidad individuales (de estos descriptores) permite definir la calidad de un borde, por lo que, en la Tabla 1 se presentan algunas formulaciones para medir estos atributos (Guzmán, 2013).

La detección de bordes por distintas técnicas se utiliza para estimar la visibilidad atmosférica; una de ellas es la extracción de altas frecuencias espaciales con filtros pasa-altos (Luo et al., 2005; Sun et al., 2007) que sobre una imagen digital en grises $f(x, y)$ de $\mathrm{MxN}$ pixeles se realiza así (ver Fig. 2): primero se construye el 
espectro de frecuencia $F(u, v)$ aplicando la transformada de Fourier (1), y luego, se multiplica el espectro por el filtro pasa-altos $H(u, v)$, (2), para finalmente, aplicar la transformada inversa de Fourier (3) y así obtener la imagen filtrada que contiene los bordes. Posteriormente sobre la imagen filtrada se define el índice de visibilidad como el promedio de las intensidades de gris (o brillo) de la imagen.

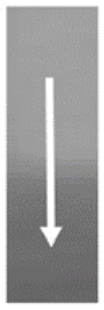

a)

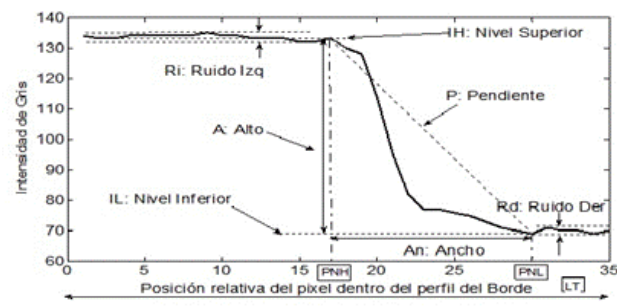

b)

Fig. 1. a) Borde: la flecha indica la trayectoria del perfil del borde, b) Perfil de borde: Intensidad de gris versus Posición relativa de cada pixel. Fuente: Autores

Tabla 1. Descriptores de cada perfil del borde. Fuente: Autores

\begin{tabular}{|c|c|c|}
\hline Descriptor & Formulación & Variables \\
\hline Alto & $A=\left(I_{H}-I_{L}\right) / 2^{8}$ & $\begin{array}{l}\text { A: Alto del borde } \\
I_{H}: \text { Intensidad de gris del nivel superior } \\
\text { del borde } \\
I_{L}: \text { Intensidad de gris del nivel inferior } \\
\text { del borde }\end{array}$ \\
\hline Ancho & $A n=\frac{\left|\mathrm{P}_{\mathrm{NL}}-\mathrm{P}_{\mathrm{NH}}\right|}{\mathrm{L}_{\mathrm{t}}}$ & $\begin{array}{l}A n: \text { Ancho del borde } \\
P_{N L}: \text { Posición pixel nivel inferior } \\
P_{N H}: \text { Posición pixel nivel superior } \\
L_{t}: \text { Longitud del perfil }\end{array}$ \\
\hline Pendiente & $P=\left(\frac{A}{A n}\right) *\left(\frac{1}{\max P}\right)$ & $\begin{array}{l}P \text { : Pendiente del borde } \\
\operatorname{maxP}: \text { Pendiente máxima entre los } 12 \\
\text { perfiles considerados en la imagen }\end{array}$ \\
\hline Ruido & $\begin{array}{l}R=\frac{S_{I}}{2^{8} * \max R} \\
S_{I}=\frac{S_{i z q}}{2}+\frac{S_{\text {der }}}{2}\end{array}$ & $\begin{array}{l}R \text { : Ruido del borde } \\
S_{i z q}: \text { Desviación estándar respecto al } \\
\text { nivel superior } \\
S_{d e r}: \text { Desviación estándar respecto al } \\
\text { nivel inferior } \\
\text { maxR: Máximo valor del ruido entre los } \\
12 \text { perfiles considerados }\end{array}$ \\
\hline
\end{tabular}


Guzmán \& Restrepo / Caracterización de Bordes y Contrastes en una Imagen Digital ante la Variación de Parámetros de los Filtros Pasa-Altos usados en la Estimación de Visibilidad Atmosférica

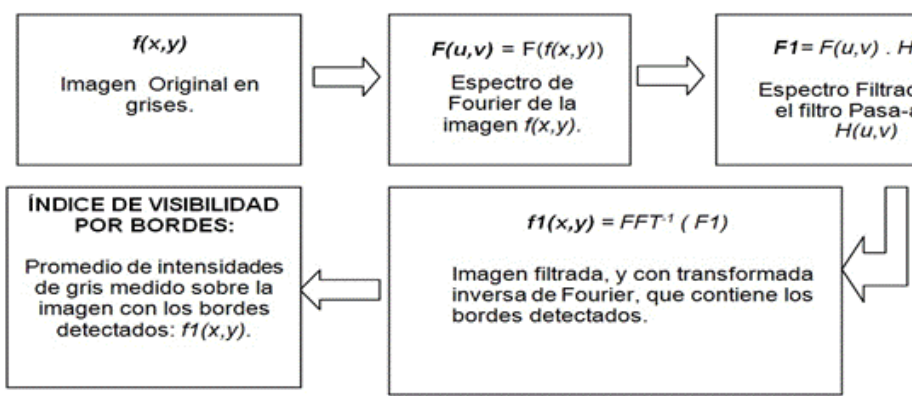

Fig. 2. Índices de visibilidad por detección de bordes mediante la extracción de altas frecuencias de la imagen con filtros pasa-altos. Fuente: Autor

$$
\begin{aligned}
& F(u, v)=\frac{1}{M N} \sum_{x=0}^{M-1} \sum_{y=0}^{N-1} f(x, y) \exp \left[-j 2 \pi\left(\frac{u x}{M}+\frac{v y}{N}\right)\right] \\
& F(u, v)=F(u, v) \cdot H(u, v) \\
& f(x, y)=\sum_{u=0}^{M-1} \sum_{v=0}^{N-1} F(u, v) \exp \left[j 2 \pi\left(\frac{u x}{M}+\frac{v y}{N}\right)\right]
\end{aligned}
$$

Los filtros pasa-altos $H(u, v)$ utilizados en las mediciones de visibilidad son: el Ideal (4), el Gaussiano (5), el HomomórficoGaussiano (6):

Filtro Ideal:

$$
H(u, v)=\left\{\begin{array}{ll}
0 & D(u, v) \leq D_{0} \\
1 & D(u, v)>D_{0}
\end{array}\right\}
$$

Filtro Gaussiano:

$$
H(u, v)=1-e^{-D^{2}(u, v) / 2 D_{0}^{2}}
$$

Homomórfico-Gaussiano: $\quad H(u, v)=\left(\gamma_{H}-\gamma_{L}\right)\left[1-e^{-c\left(\frac{D^{2}(u, v)}{D_{0}{ }^{2}}\right)}\right]+\gamma_{L}$

En (4) a (6): Las coordenadas $(u, v)$ representan frecuencias espaciales, $D$ es la distancia desde el pixel $(u, v)$ hasta el punto central del filtro $H(u, v)$; Do es el radio de corte; $c$ es el parámetro que controla la pendiente del filtro; y los parámetros $Y H$ e $Y L$ son el 
valor máximo y mínimo del filtro. La Fig. 3 muestra las representaciones bidimensionales de los filtros. Allí se pueden observar, además de la forma de sus secciones transversales, la definición de los parámetros (Do, YH, YL, c) que controlan la forma de esas secciones; según el valor de estos parámetros, los filtros $H(u, v)$ pasa-altos pueden eliminar o reducir la magnitud de la frecuencia cero del espectro de Fourier, obteniendo detección de los bordes en el primer caso y, realce de la imagen en el segundo caso (Yang et $a l ., 2005)$.
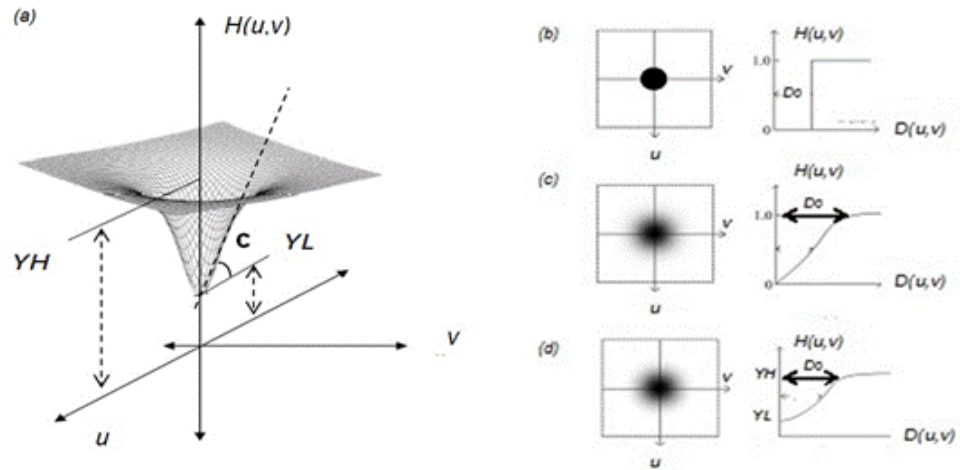

Fig. 3. a) Filtro H(u,v) Pasa-altos Homomórfico-Gaussiano, b) Ideal con radio de corte $=$ Do, c) Gaussiano con radio de corte $=$ Do, d) Homomórfico-Gaussiano con parámetros: Do, YH, YL, c. Fuente: Autores

En este trabajo, para caracterizar el comportamiento de los bordes y los contrastes frente a los diferentes filtros pasa-altos, se emplea la siguiente estrategia que se muestra en la Fig. 5: se construye una imagen sintética en grises (Fig. 4a) de 128x128 pixeles con 256 niveles de intensidad diferentes, con regiones rectangulares de diferente valor de contraste respecto a su fondo (entre el $1 \%$ y el $100 \%$, calculado según la definición de Weber en WMO-No 8, 2008), y que tiene un promedio de intensidades de gris igual a 190,31; esta imagen se filtra usando los pasa-altos resultantes al variar los parámetros $(D o, Y H, Y L, c)$, y luego se evalúa la calidad de los bordes obtenidos (ver Fig. 5). 
(a)

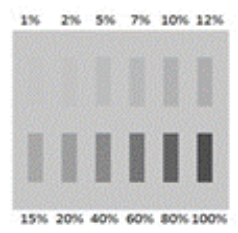

(b)

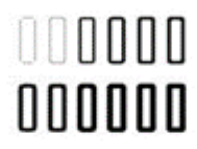

(c)

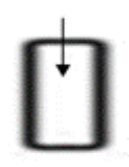

Fig. 4. a) Imagen sintética con diferentes contrastes y con Promedio del brillo = 190,31, b) Imagen filtrada con pasa-altos (en negativo), c) Borde superior de todas las regiones donde se toman los perfiles de borde. Fuente: Autores

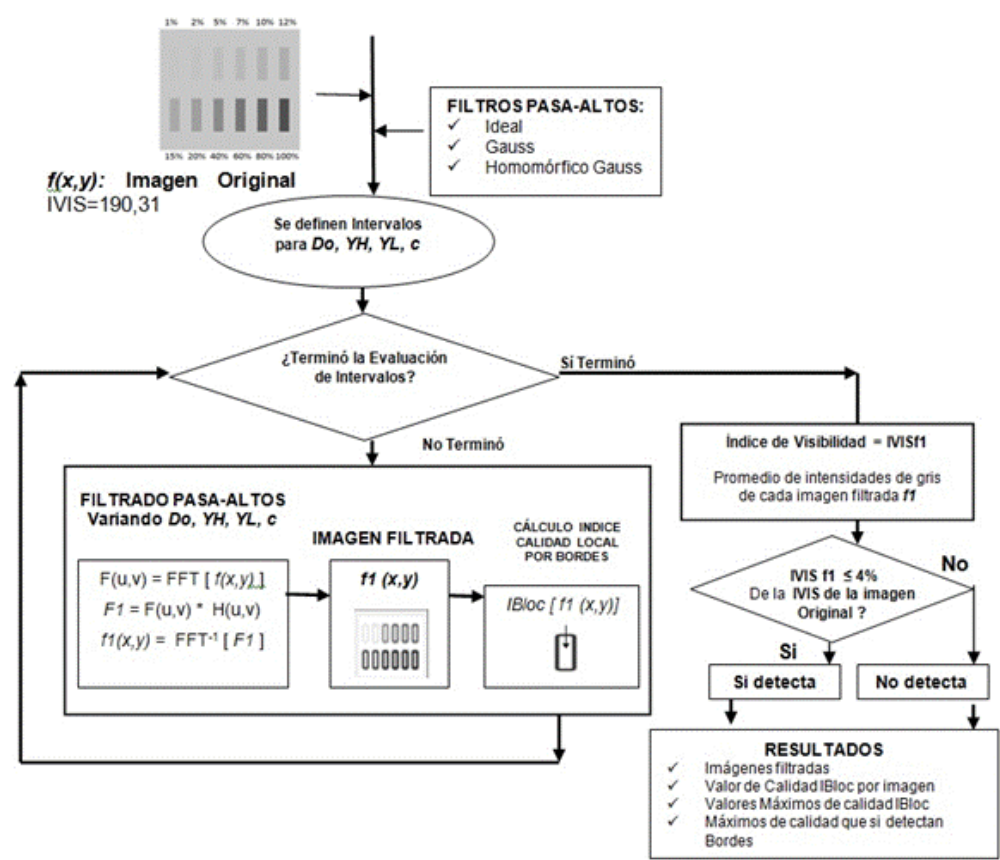

Fig. 5. Estrategia para caracterizar los contrastes y bordes obtenidos en la imagen filtrada. Fuente: Autores

Se pretende definir cuáles son los valores de los parámetros (Do, $Y H, Y L, c)$, que consiguen la mayor calidad de los bordes en la imagen sintética filtrada; puesto que la visibilidad se mide como el promedio de las intensidades de gris (o brillo), ese promedio se verá afectado con los cambios de alto, ancho, pendiente, y ruido, de los bordes obtenidos con el filtrado; por lo tanto, la mayor calidad 
en los bordes detectados (con el proceso de filtrado) permitirán estabilizar en trabajos futuros la precisión de la medida de visibilidad desde imágenes.

La evaluación de los bordes se logra con la aplicación de un índice de calidad local por bordes IBloc (Guzmán \& Restrepo, 2012) sobre el borde superior de cada uno de las regiones rectangulares (ver Fig. 4c). Este índice considera que la calidad del borde aumenta cuando: el alto y la pendiente aumentan, el ancho y el ruido disminuyen; y realiza ponderaciones para cada uno de los descriptores considerados, que luego se suman (7). Posteriormente, se suman los IBloc de todas las regiones y se obtiene así el valor de calidad para cada imagen filtrada.

IBloc $_{\text {región }}=A * 25 \%+B * 25 \%+C * 25 \%+D * 0.25 \%$

Donde $A$ corresponde al mayor alto, $B$ corresponde a menor ancho, $C$ a mayor pendiente, $D$ a menor ruido.

\section{RESULTADOS Y DISCUSIÓN}

\subsection{Filtro Ideal}

Los resultados obtenidos con el filtro Ideal, al variar el radio de corte $(D o)$, pueden verse en la Fig. 6, donde se incluyen algunas imágenes filtradas. En esta figura, se evidencia que todos los valores del radio de corte $(D o)$ no logran la detección de los bordes: por ejemplo los valores cercanos a $D o=1$. Para determinar esto automáticamente, se considera que la detección de los bordes ocurre en las imágenes filtradas cuyo promedio de intensidades de gris no supera el $4 \%$ del promedio de intensidades de la imagen original sin filtrar; y que no se consigue la detección de los bordes cuando la imagen filtrada supera este porcentaje.

También se observa que el procesamiento de la imagen logra obtener información de los bordes de todos los rectángulos considerados, pero al aumentar el $D o$ se van haciendo imperceptibles para el ojo humano, inicialmente los bordes correspondientes a las regiones con los menores contrastes y luego los bordes correspon- 
dientes a las regiones de contrastes mayores. Estas anotaciones resultan interesantes cuando se considera que el sistema visual humano tiene como límite inferior de percepción el $2 \%$ de contraste (Caimi et al., 2004), o el $5 \%$ según otros investigadores (WMONo 8, 2008).

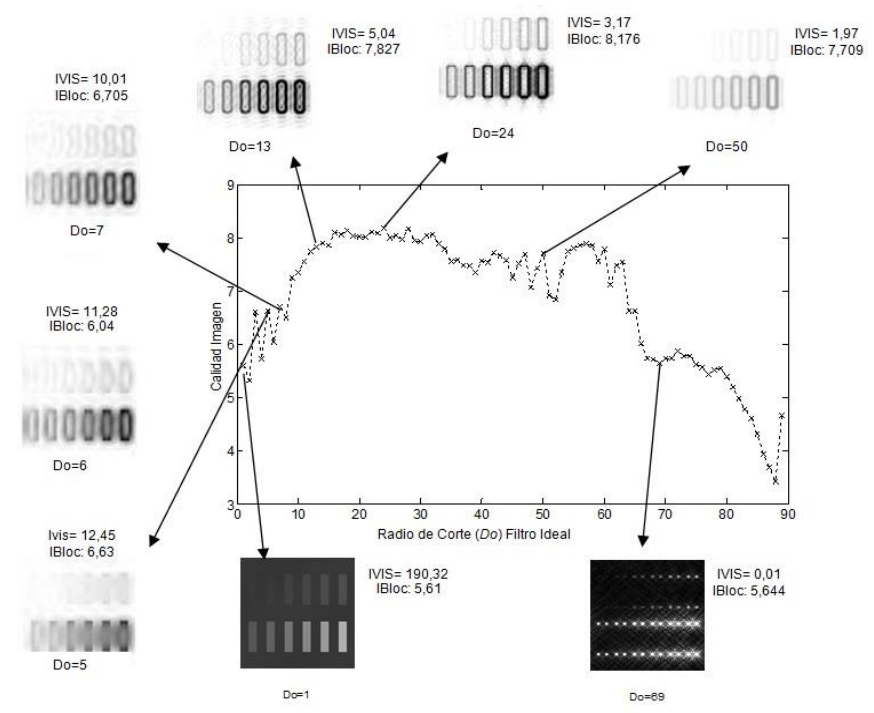

Fig. 6. Calidad (IBloc) de la imagen filtrada con Ideal vrs Radio de corte (Do). Las imágenes en formato negativo, excepto $\mathrm{Do}=69$. Fuente: Autores

También se muestra, en la Tabla 2 y en la Fig. 6, que el filtro Ideal introduce artefactos adicionales que no estaban presentes en la imagen sintética original antes del filtrado; artefactos que en el índice de calidad por bordes IBloc se miden como el ruido del borde. Estos artefactos adicionales afectan la precisión de la medida de visibilidad, puesto que los índices de visibilidad se calculan como el promedio de intensidades de gris en la imagen filtrada.

Se selecciona desde la Fig. 6, como parámetro para el filtro Ideal: $D o=24$ pixeles, equivalente al $18,75 \%$ del tamaño de la imagen. Porque la imagen filtrada obtiene el mayor valor de calidad por bordes en este filtro: $I B l o c=8,176$; y además, logra la detección de los bordes de las regiones, lo que se comprueba automáticamente con el valor del promedio de brillo (IVIS) en la ima- 
gen filtrada que no supera el $4 \%$ del promedio de brillo en la imagen original sin filtrar.

Tabla 2. Detalles de la imagen sintética filtrada con Ideal. Fuente: Autores

\begin{tabular}{|c|c|c|c|c|}
\hline Do & IBloc & Positivo & Detalle Positivo & Índice de Visibilidac \\
\hline 1 & 7,354 & & & 5,75 \\
\hline 24 & 8,176 & I & & 3,17 \\
\hline
\end{tabular}

\subsection{Filtro Gaussiano}

Los resultados obtenidos, para el filtro Gaussiano al variar el radio de corte $(D o)$, pueden verse en la Fig. 7 y en la Tabla 3. Donde, se evidencia que todos los radios de corte no logran la detección de los bordes: por ejemplo, las imágenes filtradas con $1 \leq D o \leq 6$ no lo logran, o lo logran parcialmente. Y se evidencia que, el filtrado con Gaussiano también introduce artefactos (o ruido) en la imagen filtrada que no estaban presentes en la imagen original.

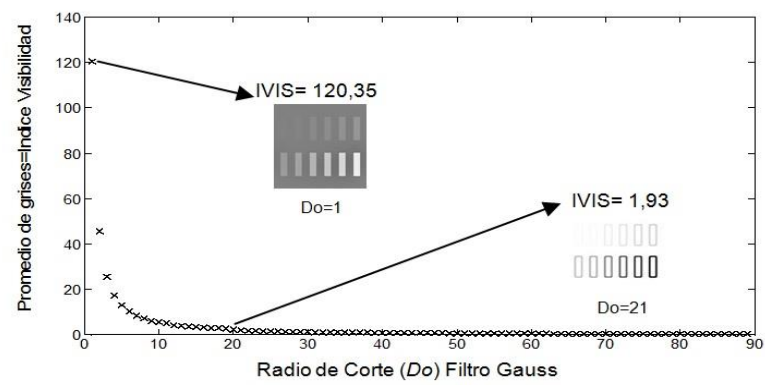

Fig. 7. Índice de visibilidad con Gaussiano (IVIS) vrs Radio de corte (Do). Imágenes en negativo. Fuente: Autores

En la Fig. 8, el $D_{o}=1$ muestra el mayor índice de calidad $(I B l o c=8,055)$, pero presenta un brillo promedio igual a 120,35 que es mayor al $4 \%$ de la intensidad promedio de la imagen original 
Guzmán \& Restrepo / Caracterización de Bordes y Contrastes en una Imagen Digital ante la Variación de Parámetros de los Filtros Pasa-Altos usados en la Estimación de Visibilidad Atmosférica

sin filtrar; lo que permite indicar automáticamente que no logra la detección de los bordes. Por lo tanto, se selecciona el parámetro $D o=21$ pixeles, o $16,41 \%$, porque la imagen filtrada obtiene el mayor valor de calidad por bordes con este filtro $(I B l o c=7,837)$ que sí logra la detección de los bordes.

Tabla 3. Detalles de la imagen sintética filtrada con Gaussiano. Fuente: Autores

\begin{tabular}{lllll}
\hline Do & IBloc & Positivo & Detalle & Indice de Visibilidad \\
\hline 21 & 7,837 & Artefactos & 1,93 \\
\hline 32 & 7,707 & Artefactos & 1,10 \\
& & &
\end{tabular}

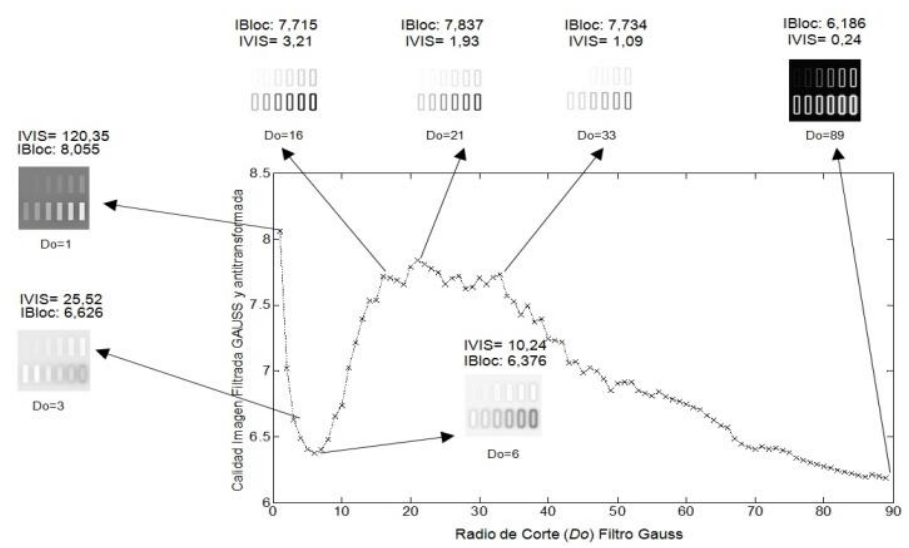

Fig. 8. Calidad (IBloc) de la imagen filtrada con Gaussiano vrs Radio de corte (Do). Las imágenes filtradas en negativo (excepto Do=89). Fuente: Autores

\subsection{Filtro Homomórfico-Gaussiano}

El filtro Homomórfico-Gaussiano contiene, además del Do, otros parámetros que cumplen diferentes funciones en el filtrado de la imagen (Liaw et al., 2010). Por ejemplo: YH permite controlar la reflectancia o altas frecuencias de la imagen, $Y L$ facilita el control de la iluminación de la imagen o las bajas frecuencias, y $c$ 
con el cual se puede manejar la pendiente del filtro. Por lo tanto, inicialmente se establece para la detección de los bordes de la imagen: $Y H=1$ para obtener todas las altas frecuencias del espectro de Fourier, y el $Y L=0$ para eliminar completamente la frecuencia cero.

Entonces con $Y H=1$ y con $Y L=0$, se comienza por variar el parámetro $c$ y observar el comportamiento de la calidad de la imagen filtrada cuando el radio de corte cambia entre $15 \leq D o \leq 35$; se escoge este intervalo porque corresponde a valores cercanos al $D o=21$ que produjo la mejor calidad con el filtro Gaussiano. Los resultados obtenidos se muestran en la Fig. 9 y en la Tabla 4.

Tabla 4. Detalle de la imagen sintética filtrada con Homomórfico-Gaussiano usan-

\begin{tabular}{|c|c|c|c|c|}
\hline Do & c & Filtrada & Detalle & Índice Visibilidad \\
\hline 21 & 0,2 & & & 1,07 \\
\hline 21 & 0,4 & & & 1,64 \\
\hline 21 & 1 & $\begin{array}{l}00, \frac{1}{0} \\
011 M\end{array}$ & $\begin{array}{lll}1 & 0 \\
- & & \\
1 & & \end{array}$ & 3,43 \\
\hline
\end{tabular}

En la Fig. 9a, se evidencia que todas las $c$ en el intervalo $0<c$ $\leq 1$ detectan bordes, aunque, las regiones correspondientes a los menores contrastes resultan casi imperceptibles para el ojo humano. También, se muestra en la Fig. 9b que cuando $c>1$ la detección de bordes es parcial, o no se detectan bordes; y en la Tabla 4, se evidencia que algunos valores de los parámetros adicionan más artefactos que otros a la imagen filtrada.

Desde la Fig. 9, para el filtro Homomórfico Gaussiano se selecciona la combinación de parámetros: $D o=21, Y H=1, Y L=0, c=1$. Porque proporciona alta calidad de imagen por bordes (IBloc $=$ $7,8373)$ y logra la detección de los bordes; ver Tabla 4 donde se 
Guzmán \& Restrepo / Caracterización de Bordes y Contrastes en una Imagen Digital ante la Variación de Parámetros de los Filtros Pasa-Altos usados en la Estimación de Visibilidad Atmosférica

muestra que su promedio de intensidades de gris es inferior al $4 \%$ del valor de la imagen original sin filtrar, y que la adición de artefactos es baja.

(a) Si detectan bordes

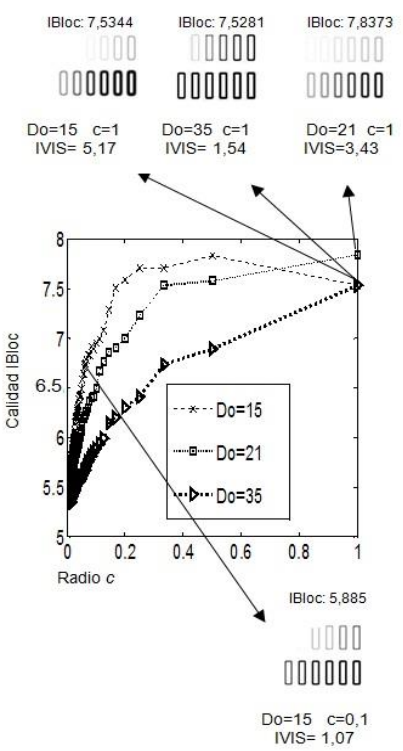

(b) No detectan bordes

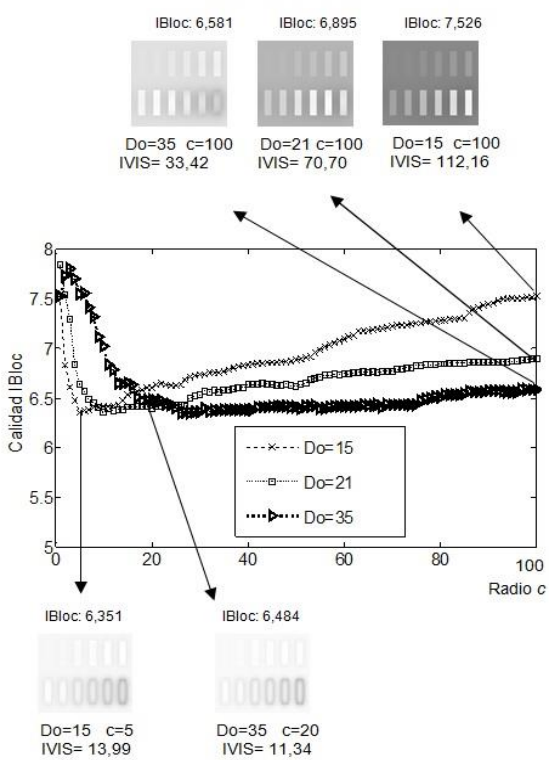

Fig. 9. Calidad (IBloc) de la imagen filtrada con Homomórfico-Gaussiano YH=1, $\mathrm{YL}=0$, vrs c. a) $0,1 \leq \mathrm{c} \leq 1$ para $15 \leq \mathrm{Do} \leq 35$, b) $1 \leq \mathrm{c} \leq 100$ para $15 \leq$ Do $\leq 35$. Todas las imágenes en negativo. Fuente: Autores

Sin embargo, considerando que la amplitud del filtro Homomórfico está relacionada con la expresión ( $Y H-Y L)$ según (3), se mantienen fijos $D o=21, c=1, Y H=1$, y se revisa el comportamiento de los bordes frente al uso de valores mayores del $Y L$, y frente a una variación más amplia del radio de corte $D o$; los resultados se muestran en la Fig. 10. En ella se observa que la mayor calidad de imagen por bordes $(I B l o c=9,087)$ se obtiene con $D o=30, c=1, Y H=1$, $Y L=0,9$, y que la combinación $D o=30, c=1, Y H=1, Y L=0,5$ produce también un valor elevado de calidad. Sin embargo, al revisar las imágenes filtradas se observa que estas combinaciones aunque realzan la calidad no logran la detección de los bordes; lo cual puede verificarse al observar sus valores del promedio de intensi- 
dad de gris en la imagen filtrada. En cambio, el máximo de calidad IBloc $=7,8373$ obtenido con $D o=21, c=1, Y H=1, Y L=0$, sí logra la detección de los bordes.

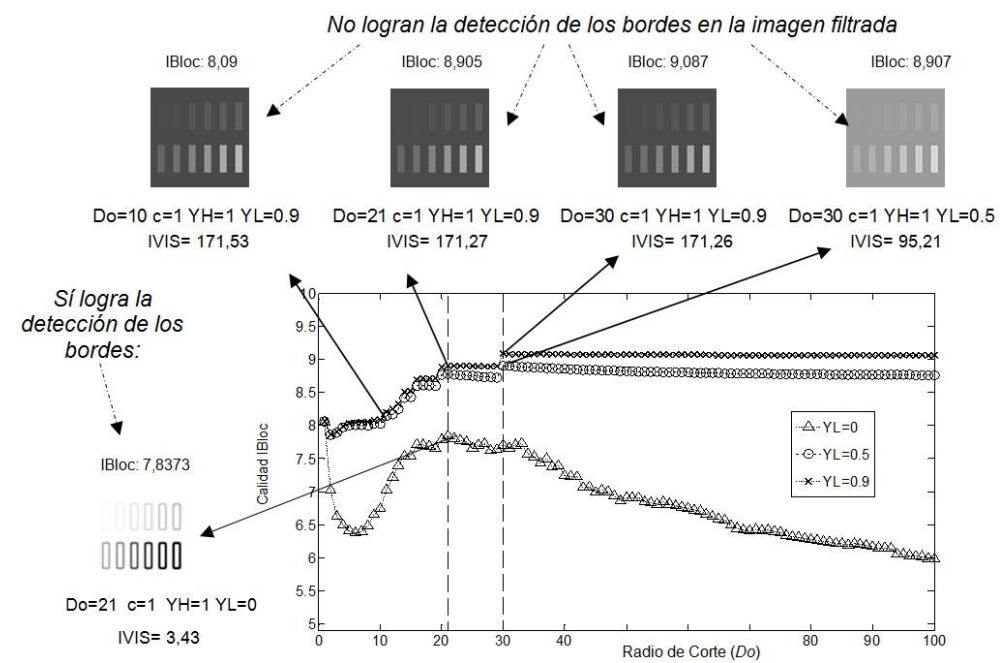

Fig. 10. Filtro Homomórfico Calidad (IBloc) vrs Radio de corte (Do), para c=1, $\mathrm{YH}=1$. Con $0 \leq \mathrm{YL} \leq 0,9$ Las imágenes en negativo. Fuente: Autores

Por lo tanto, para la aplicación considerada, los parámetros seleccionados para el filtro Homomórfico-Gaussiano son: $D o=21$ pixeles o $16,41 \%, c=1, Y H=1, Y L=0$. Porque detectan los bordes, ofrecen buena calidad de bordes, y también introducen menos ruido o artefactos adicionales en la imagen filtrada.

\subsection{Parámetros Seleccionados para los Filtros Pasa-altos}

En la Tabla 5 se muestra el resumen con los parámetros seleccionados para todos los filtros considerados. El radio de corte $(D o)$ se muestra en porcentaje con respecto al número de pixeles que representan la imagen. 
Guzmán \& Restrepo / Caracterización de Bordes y Contrastes en una Imagen Digital ante la Variación de Parámetros de los Filtros Pasa-Altos usados en la Estimación de Visibilidad Atmosférica

Tabla 5. Resumen de los parámetros seleccionados para los filtros Pasa-Altos. Fuente: Autores

\begin{tabular}{|c|c|c|c|c|c|}
\hline \multirow{5}{*}{$\begin{array}{l}\text { Imagen original sin } \\
\text { filtrar } \\
16 \times 2 \% 5 \% 7610 \% 126\end{array}$} & $\begin{array}{l}\text { Filtros pasa- } \\
\text { altos }\end{array}$ & Do & $\begin{array}{c}\text { Negativo de } \\
\text { Imagen Filtrada }\end{array}$ & $\begin{array}{l}\text { Îndice de } \\
\text { Visibilidad }\end{array}$ & IBloc \\
\hline & IDEAL & $18,75 \%$ & & 3,17 & 8,176 \\
\hline & GAUSS & $16,41 \%$ & & 1,93 & 7,8370 \\
\hline & $\begin{array}{l}\text { HOMOMÓRFICO- } \\
\text { GAUSS }\end{array}$ & & & & \\
\hline & $\underset{c=1}{Y H=1} \quad Y L=0$ & $16,41 \%$ & & 3,43 & 1,80313 \\
\hline
\end{tabular}

\section{CONCLUSIONES}

Se caracteriza el comportamiento de los bordes y los contrastes, ante la variación de los parámetros de los filtros pasa-altos que se utilizan en la medición de visibilidad atmosférica: aplicando los filtros del tipo Ideal, Gaussiano, y Homomórfico-Gaussiano, sobre una imagen digital sintética que contiene regiones con diferentes contrastes. Luego, se define un índice de calidad para los bordes y se utiliza para comparar los bordes obtenidos (sobre la imagen filtrada) cuando se varían los parámetros (Do, YH, YL, c) de cada filtro considerado.

Los resultados muestran que tanto al cambiar el tipo de filtro pasa-altos como al variar sus parámetros, las características del borde tales como ancho, altura y pendiente cambian; también cambia la cantidad de ruido que se adiciona a la imagen filtrada (artefactos que no estaban presentes en la imagen original) y se observa que estos cambios resultan distintos para las regiones con diferentes contrastes. Los resultados también evidencian que todas las combinaciones de los parámetros (Do, YH, YL, c) no son exitosas en la detección de los bordes, que son requeridos para las estimaciones de visibilidad desde imágenes. Así que, empleando 
un criterio para definir automáticamente si esto se logra, se descartan aquellas combinaciones de parámetros que no consiguen la detección de los bordes en la imagen filtrada.

Todo lo anterior se traduce en que la selección del tipo de filtro, tanto como la selección de sus parámetros, resulta en bordes diferentes; lo que afecta la precisión de la medida de visibilidad atmosférica que se define como el promedio de brillo en la imagen digital filtrada. También se encontró en este trabajo que, el índice de calidad definido para los bordes alcanza valores máximos con ciertas combinaciones de los parámetros correspondientes a los filtros pasa-altos. Así que para todos los filtros considerados, con estos máximos de calidad, se establecieron los parámetros que disminuyen las situaciones que deterioran la calidad de los bordes en las imágenes filtradas.

Esto se traduce en que al mejorar la calidad de los bordes, con el uso de los parámetros establecidos aquí para los filtros pasaaltos, se reduce la distorsión de las medidas de visibilidad desde imágenes. Lo que permitirá, en estudios futuros, estabilizar la precisión de las medidas de visibilidad basadas en la transformada de Fourier. Como estudio futuro, también se propone utilizar los filtros pasa-altos establecidos en este trabajo para estimar visibilidad atmosférica y luego establecer correlaciones con mediciones realizadas con equipos transmisómetros, y/o nefelométricos.

\section{AGRADECIMIENTOS}

Los autores expresan su agradecimiento al Instituto Tecnológico Metropolitano por el financiamiento de este trabajo, enmarcado dentro del proyecto de investigación código PM11103 y denominado: Metodología para Medir Visibilidad orientada a la detección de Eventos Atmosféricos usando análisis digital de imágenes.

\section{REFERENCIAS}

Bäumer, D., Versick, S. \& Vogel, B. (2008). Determination of the visibility using a digital panorama camera. Atmospheric Environment, 42(11), 2593-2602. 
[140] Guzmán \& Restrepo / Caracterización de Bordes y Contrastes en una Imagen Digital ante la Variación de Parámetros de los Filtros Pasa-Altos usados en la Estimación de Visibilidad Atmosférica

Caimi, F. M., Kocak, D. M., \& Justak, J. (2004). Remote visibility measurement technique using object plane data from digital image sensors. Proceedings of the Geoscience and Remote Sensing Symposium, 5, 3288-3291.

Guzmán, M. (2013). Metodología para medir visibilidad orientada a la detección de eventos atmosféricos usando análisis digital de imágenes. Tesis de Maestría no publicada dirigida por Alejandro Restrepo Ph.D. Maestría en Automatización y Control Industrial. Instituto Tecnológico Metropolitano, Medellín, Colombia.

Guzmán, M., \& Restrepo, A. (2012). The image quality in the measurement of atmospheric visibility from Contrast Indices and Edges. Medellín, XVII Symposium of Image, Signal Processing and Artificial Vision (STSIVA), 196-201.

Liaw, J. J., Lian, S. B., Huang, Y. F. \& Chen, R. C. (2010). Using sharpness image with Haar function for urban atmospheric visibility measurement. Aerosol and Air Quality Research, 10, 323-330.

Luo, Ch. H., Wen, Ch. Y, Yuan, Ch. S., \& Liaw, J.J. (2005). Investigation of urban atmospheric visibility by high-frequency extraction: Model development and field test. Atmospheric Environment, 39, 2545-2552.

Sun, Y. C., Liaw, J. J. \& Luo, Ch. H. (2007). Measuring Atmospheric Visibility Index by Different High-pass operations. Taiwan, Computer Vision Graphics and Image Processing Conference (CVGIP), 423-428.

Töpfer, S. C. N. \& Linss, G. (2007). Quality measures for optical probing in Optical Coordinate Metrology. Measurements Science Review, 4(7), Section 3, 51-54.

WMO-No 8 (2008). Guide to meteorological instruments and methods of observation. World Meteorological Observations. 7th ed. Secretariat of the WMO, Geneva, Switzerland.

Xie, L., Chiu, A. \& Newsam, S. (2008). Estimating atmospheric visibility using general purpose cameras. Las Vegas, Nevada, Advances in visual computing: $4^{\text {th }}$ International Symposium, (ISVC), 356-367.

Yang, C.C. (2005). Improving the sharpness of an image with non-uniform illumination. Optical Laser Technologies, 37, 235-238. 